\title{
Rapidly Visible-light-mediated Photogelations for One-step Engineering Multifunctional Tough Hydrogel Tubes
}

\author{
Wenqing Xiao, Ping Zhang, Hongqiu Wei and You Yu*
}

Key Laboratory of Synthetic and Natural Functional Molecule Chemistry of the Ministry of Education, College of Chemistry and Materials Science, Northwest University, Xi' an China, 710000.

Corresponding Email: yuyou@nwu.edu.cn

\section{Chemicals}

Tris(2,2'-bipyridyl)dichlororuthenium(II) (Ru(II), Acros), poly(ethylene glycol) ( 20,000 Da, SigmaAldrich), gelatin (Type A, 300 g Bloom from porcine skin, Sigma-Aldrich), polyquaternium-10 (JR-400, 1.6-2.1 N\%), polylactic acid (4032D, NatureWorks), acrylamide (TCI) and other chemicals (Sinopharm Chemical Reagent Co. Ltd.) were bought and used without further purification.

\section{One-step preparation of bulk, 3D and tubular hydrogels in seconds}

A mixture of gelatin and Q10 was vigorously stirred in hot water to form a transparent solution. After cooling to room temperature, AAm and MBA were added and stirred for $10 \mathrm{~min}$. Then, Ru(II) and persulfate salts were added and stirred for another $10 \mathrm{~min}$ until the persulfate salts were fully dissolved. Next, the solution was centrifuged for $2 \mathrm{~min}$ at $13000 \mathrm{rpm}$ to remove air bubbles and injected into a polytetrafluoroethylene mold $(15 \mathrm{~mm} \times 40 \mathrm{~mm} \times 2 \mathrm{~mm})$. Finally, bulk hydrogels were obtained when exposing the solution to visible light irradiation $(452 \mathrm{~nm})$ for 60 seconds. Notably, to make tough hydrogel patterns, a shadow mask was placed on the top of this solution prior to irradiation. After that, the unreacted places were gently rinsed by deionized water for several times to develop patterned hydrogels.

As for the 3D hydrogel structures, the solution was firstly injected into black syringes and then extruded from the single or coaxial nozzles. It worth noting that the visible light irradiation was immediately applied to trigger the gelation process. The pyramid- and finger- shape hydrogels were obtained by a single nozzle. The fabrication procedure of tubular hydrogels was similar to 3D printing of hydrogels, but the coaxial nozzle was used instead of the single nozzle. In order to make hydrogel tubes, a highly viscostic aqueous ink of Q10 (10 wt\%) was used as the sacrificial flow in the inner channel. When preparing functional tubes, the materials, i.e., $\mathrm{Fe}_{3} \mathrm{O}_{4} / \mathrm{PLA}(1: 3)$ in dichloride $(25 \mathrm{wt} \%)$, rhodamine B/Q10 (1:40) in ethylene glycol (10 wt\%) and EDOT/Ru(II)/ $\mathrm{Na}_{2} \mathrm{~S}_{2} \mathrm{O}_{8} / \mathrm{Q} 10$ (1:0.008:4:8) in water (30 $\mathrm{wt} \%$ ) were respectively injected into syringes to replace the sacrificial ink of Q10. After co-spinning with 
the hydrogel precursor under visible light irradiation, the corresponding magnetic, shape-memory, fluorescent, and conductive tubes were obtained.

\section{Characterizations}

As for rheology testing, all tests were performed on Anton Paar MCR302 at room temperature with a strain of $1 \%$ at $10 \mathrm{~Hz}$. The experimental setup was shown in Figure S6. Typically, $1.6 \mathrm{~mL}$ of solution was firstly injected onto the plate (diameter of $50 \mathrm{~mm}$ ) with a gap of $1,000 \mu \mathrm{m}$. Then, the rotor slowly went down and attached the solution surface. After that, visible light was applied through the transparent quartz plate $\left(15 \mathrm{~mW} \mathrm{~cm}^{-2}\right)$, and the storage and loss modulus were recorded immediately.

All tensile tests were performed at room temperature with a speed of $50 \mathrm{~mm} \mathrm{~min}^{-1}$. Samples (40 $\mathrm{mm}$ $\times 15 \mathrm{~mm} \times 2 \mathrm{~mm}$ ) were adhered to the clampers using $3 \mathrm{M}$ tapes. The compression tests were carried out at the speed of $10 \mathrm{~mm} \mathrm{~min}^{-1}$. To evaluate the effect of salts on the mechanical properties, the as-prepared hydrogels were slowly dried at room temperature. When the weight decreased by $50 \%$, the same amount of corresponding solutions was added onto the hydrogel surfaces, following by a waiting period of $6 \mathrm{~h}$ to disperse solutions in hydrogels uniformly. After that, the samples were dried and swollen again according to the same procedure. Finally, the samples were tested in the same way as the fresh samples.

Moreover, to evaluate the adhesion property of different hydrogels, the samples $(20 \mathrm{~mm} \times 20 \mathrm{~mm} \times$ $2 \mathrm{~mm}$ ) were sandwiched between two glass slides and adhered to clampers. The force data were recorded when stretching the glass slides.

\section{Knitting hydrogel tubes to 3D networks}

To fabricate a 3D tubular network, two hydrogel tubes were first knitted together. Then, different dye solutions in ethylene glycol were injected into these tubes. Next, the hydrogel precursor of PAAm/Q10/G was infiltrated into the knitted tubes. After exposure to visible light illumination, the flexible and stretchable 3D network was obtained. 


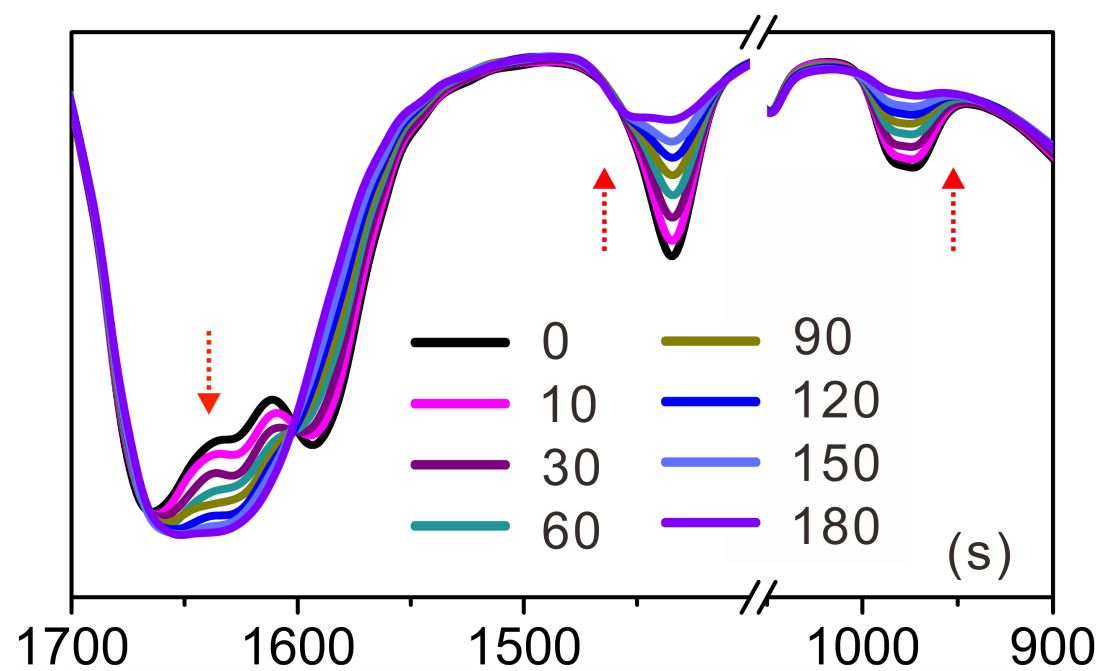

Figure S1. FT-IR characterization of the polymerization of PAAm in situ with continuous irradiation. $[\mathrm{Ru}(\mathrm{II})]=13.4 \mu \mathrm{M},\left[\mathrm{Na}_{2} \mathrm{~S}_{2} \mathrm{O}_{8}\right]=5 \mathrm{wt} \%,[\mathrm{AAm}]=30 \mathrm{wt} \%$, the irradiation intensity is $15 \mathrm{~mW} \mathrm{~cm}^{-2}$.

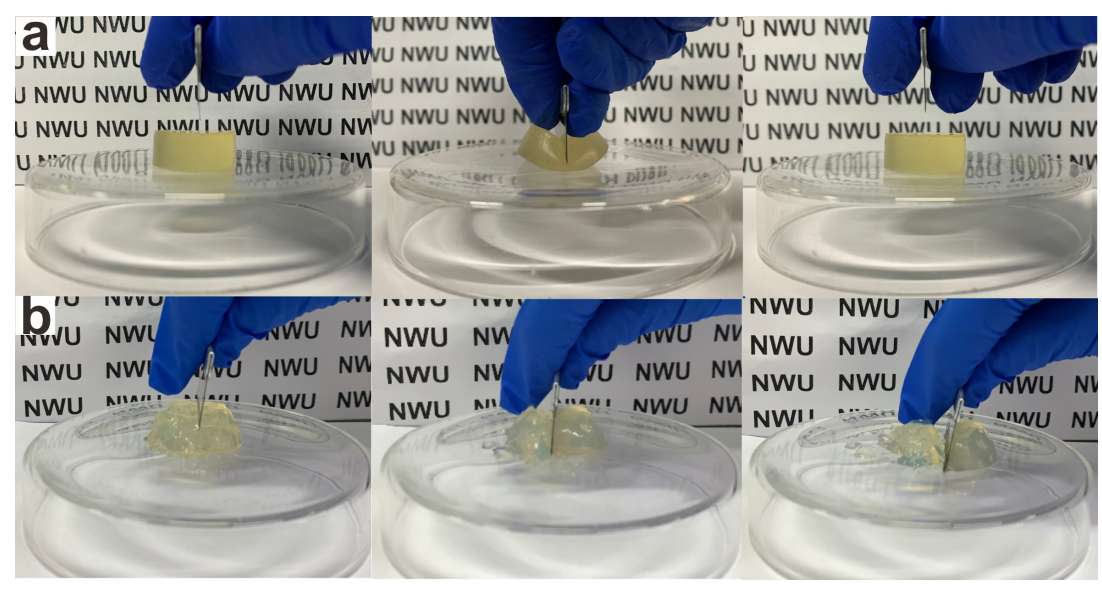

Figure S2. (a) PAAm/Q10/G and (b) pure gelatin hydrogels were cut by a razor blade.
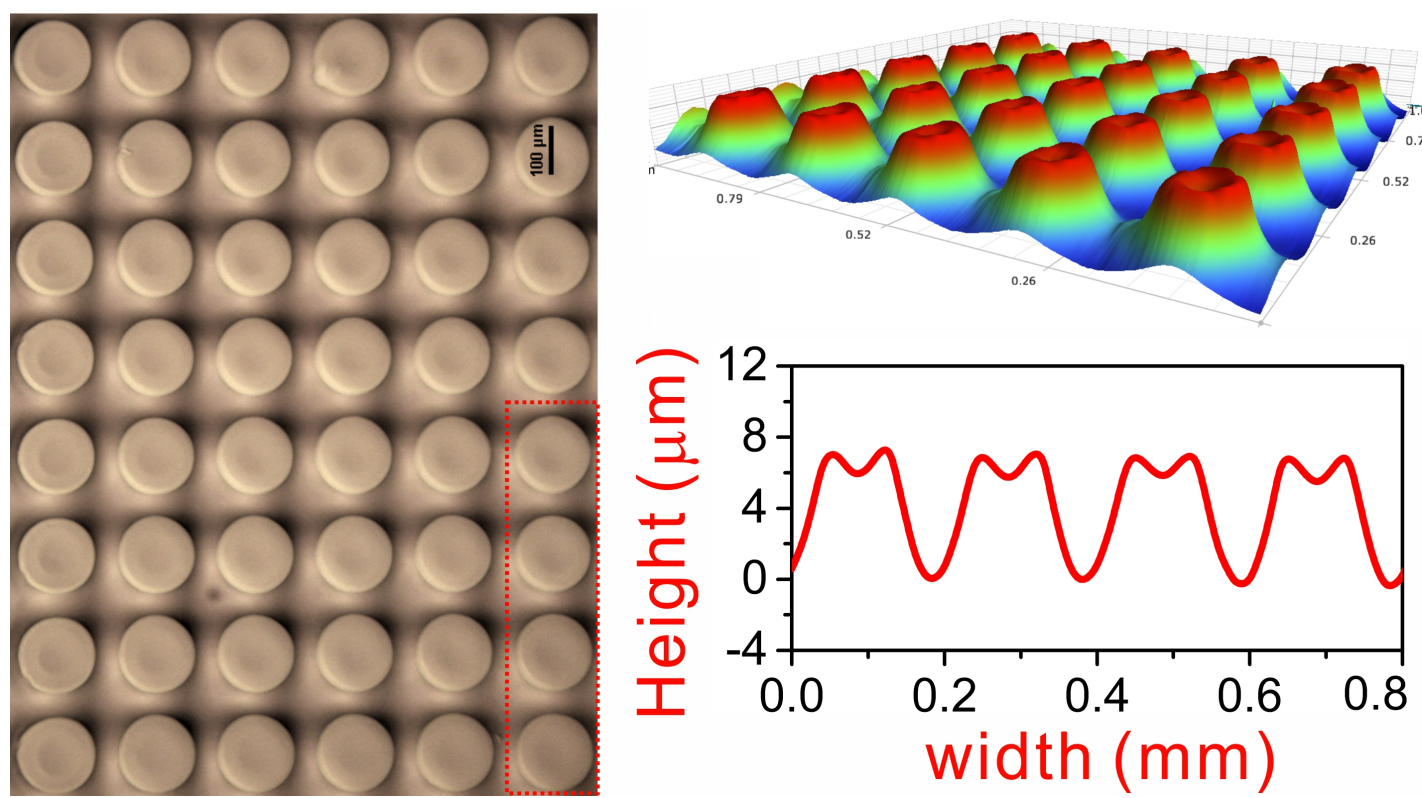

Figure S3. Surface morphology of as-prepared cylinder PAAm/Q10/G arrays. 


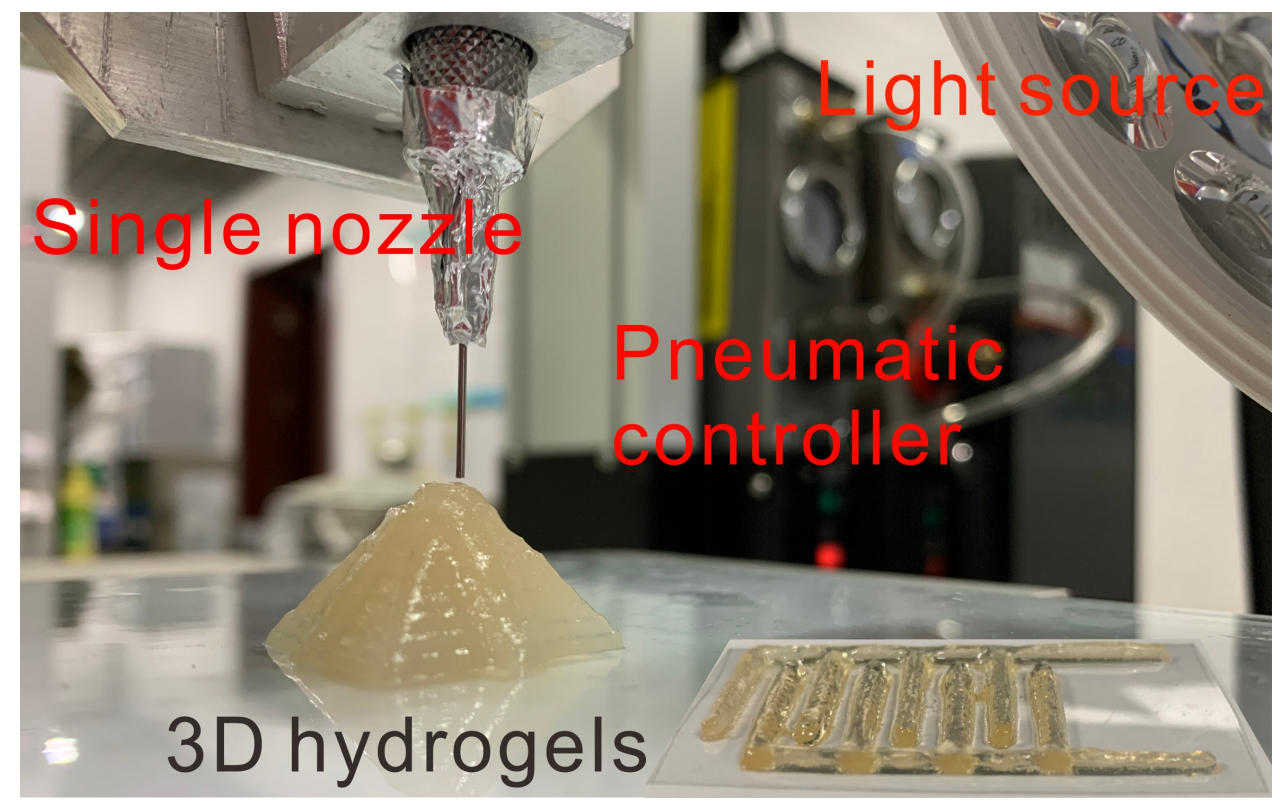

Figure S4. The 3D printing system for fabricating tough pyramid and finger-shaped hydrogels.

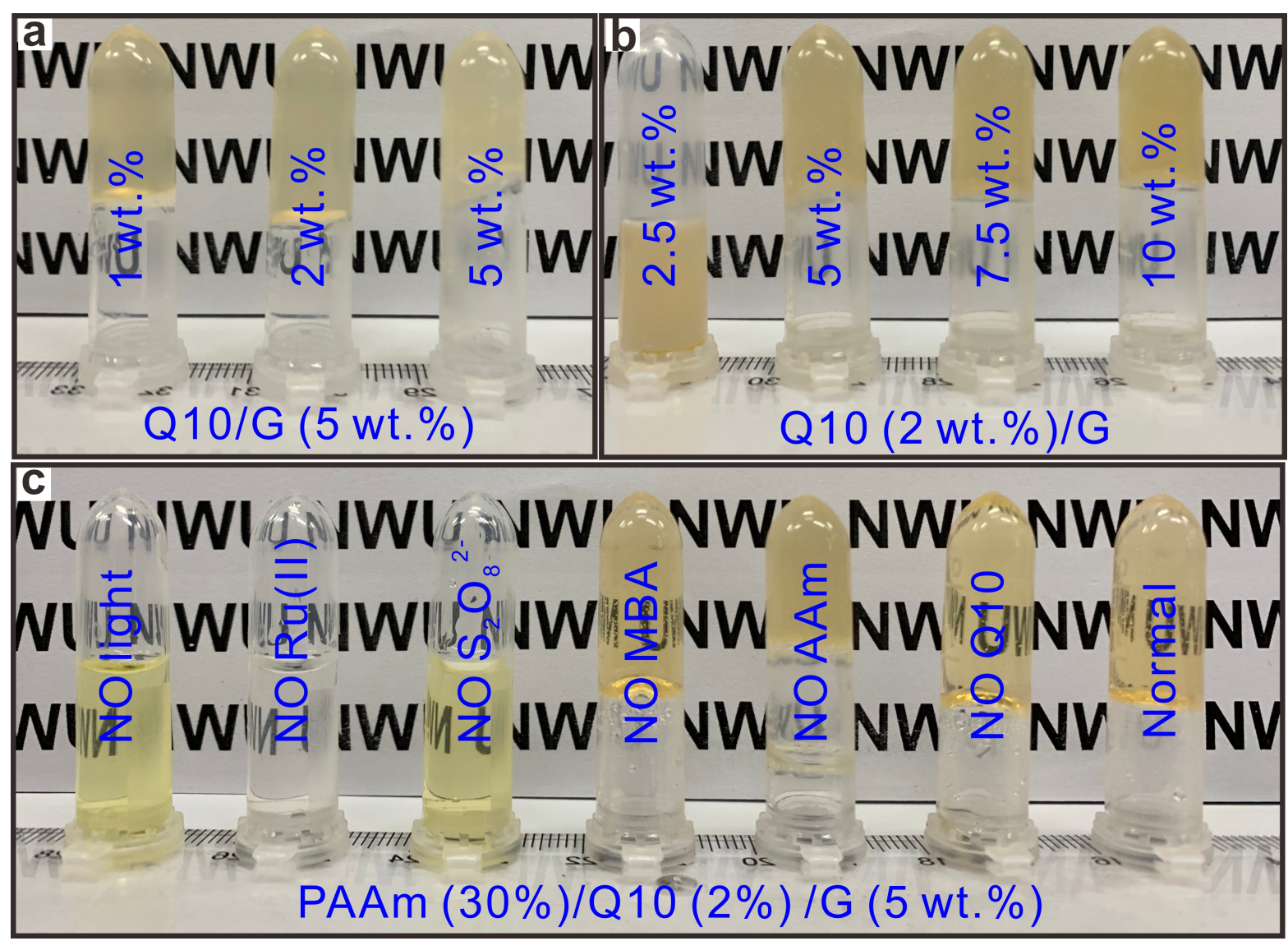

Figure S5. The effects of the concentrations of (a) Q10 and (b) gelatin on the gelation processes of Q10/G hydrogels. (c) A series of control systems for fabricating PAAm/Q10/G hydrogels. Each system lacks one component or light irradiation. 


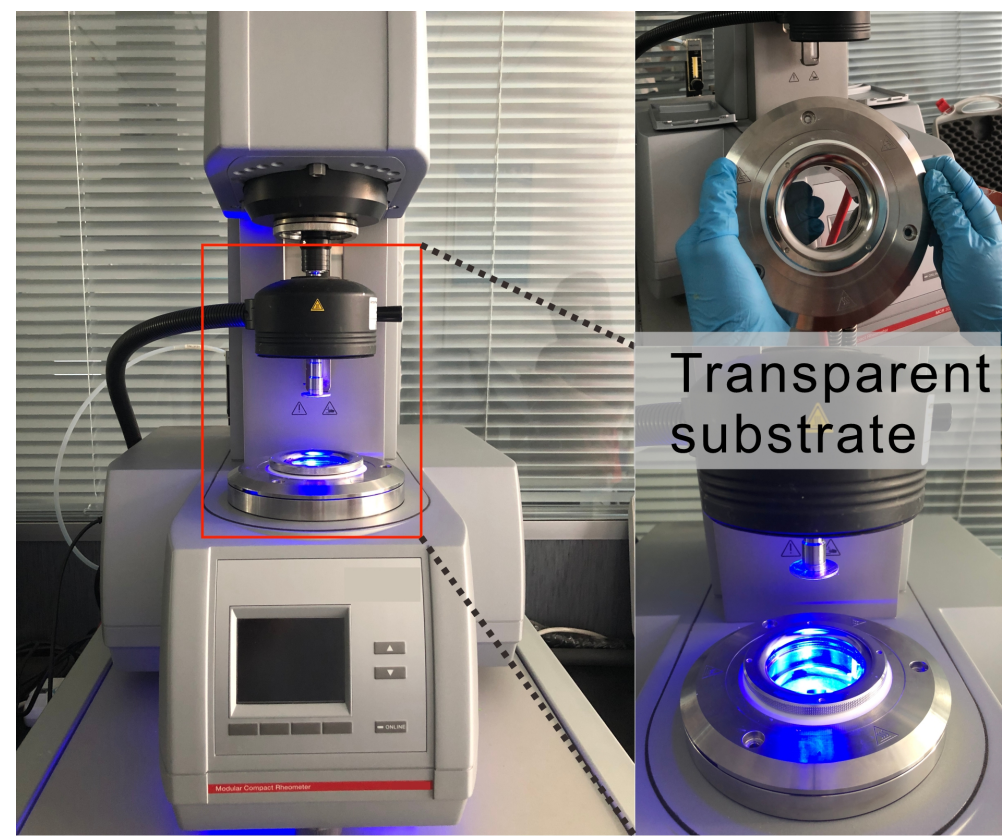

Figure S6. The experiment setup for the rheological test in this study. A typical transparent substrate was used for allowing blue light to transmit and trigger orthogonal photogelation in this case.
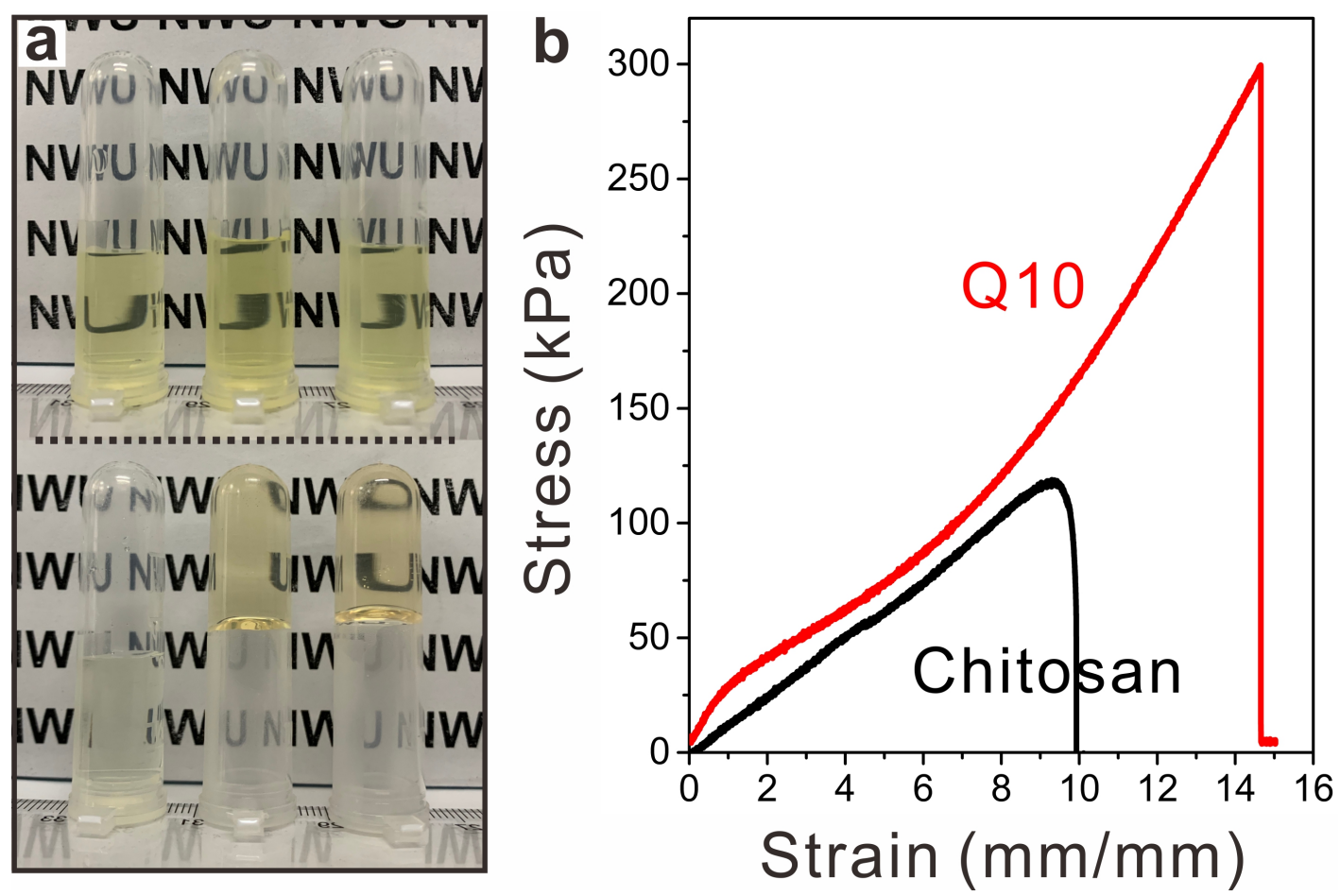

Figure S7. (a) Pure AAm, AAm/chitosan and AAm/Q10 hydrogels (left to right) before and after bluelight irradiation. $[\mathrm{Ru}(\mathrm{II})]=13.4 \mu \mathrm{M},\left[\mathrm{Na}_{2} \mathrm{~S}_{2} \mathrm{O}_{8}\right]=1 \mathrm{wt} \%,[\mathrm{AAm}]=30 \mathrm{wt} \%$, [chitosan $]=[\mathrm{Q} 10]=2 \mathrm{wt} \%, 60$ $\mathrm{s}$ irradiation at the intensity of $15 \mathrm{~mW} \mathrm{~cm}^{-2}$. (b) Stress-strain curves of $\mathrm{PAAm} / \mathrm{Q} 10 / \mathrm{G}$ and PAAm $/$ chitosan $/ \mathrm{G}$ hydrogels. $[\mathrm{MBA}]=0.03 \mathrm{~mol} \%$, [gelatin] $=5 \mathrm{wt} \%$. Other conditions are as same as that in Figure (a). 


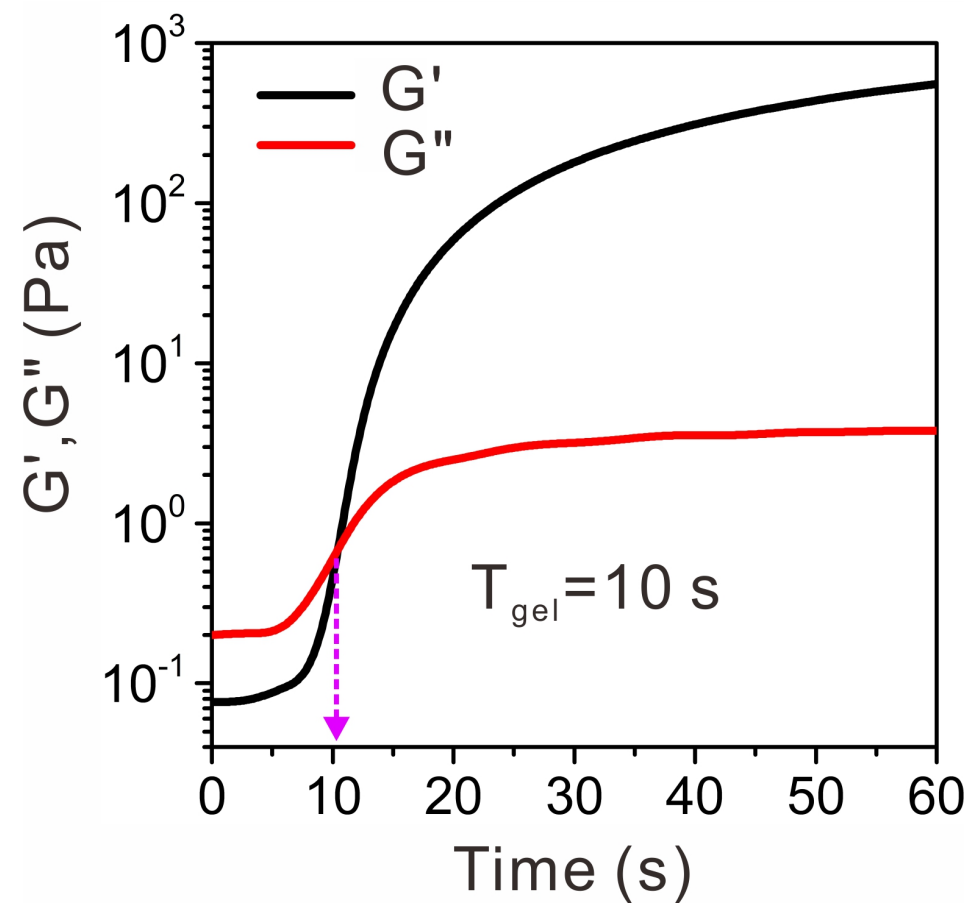

Figure S8. Rheology characterization of pure gelatin solution ( $5 \mathrm{wt} \%)$ with continuous irradiation. $[\mathrm{Ru}(\mathrm{II})]=13.4 \mu \mathrm{M},\left[\mathrm{Na}_{2} \mathrm{~S}_{2} \mathrm{O}_{8}\right]=1 \mathrm{wt} \%$, the irradiation intensity is $15 \mathrm{~mW} \mathrm{~cm}{ }^{-2}$.
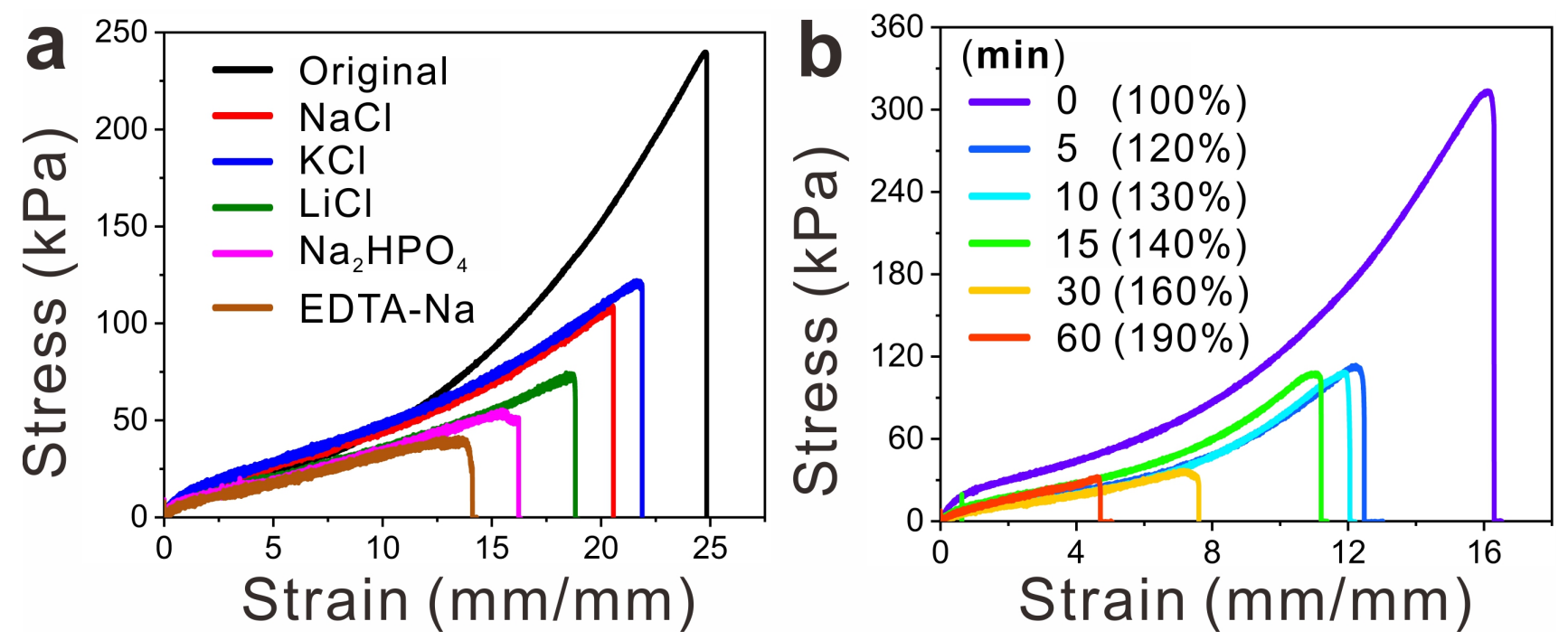

Figure S9. Mechanical tests of (a) alginate $/ \mathrm{Ca}^{2+} / \mathrm{PAAm}$ with the treatment of different salts and (b) PAAm/Q10/G with different immersing time in the water. Insets show the swelling ratios (\%) of hydrogels with corresponding immersing times. The swelling ratio is calculated by comparing the weights of swollen and original samples. Alginate $/ \mathrm{Ca}^{2+} / \mathrm{PAAm}$ hydrogels in Figure (a) are prepared according to the literature ${ }^{1}$. 

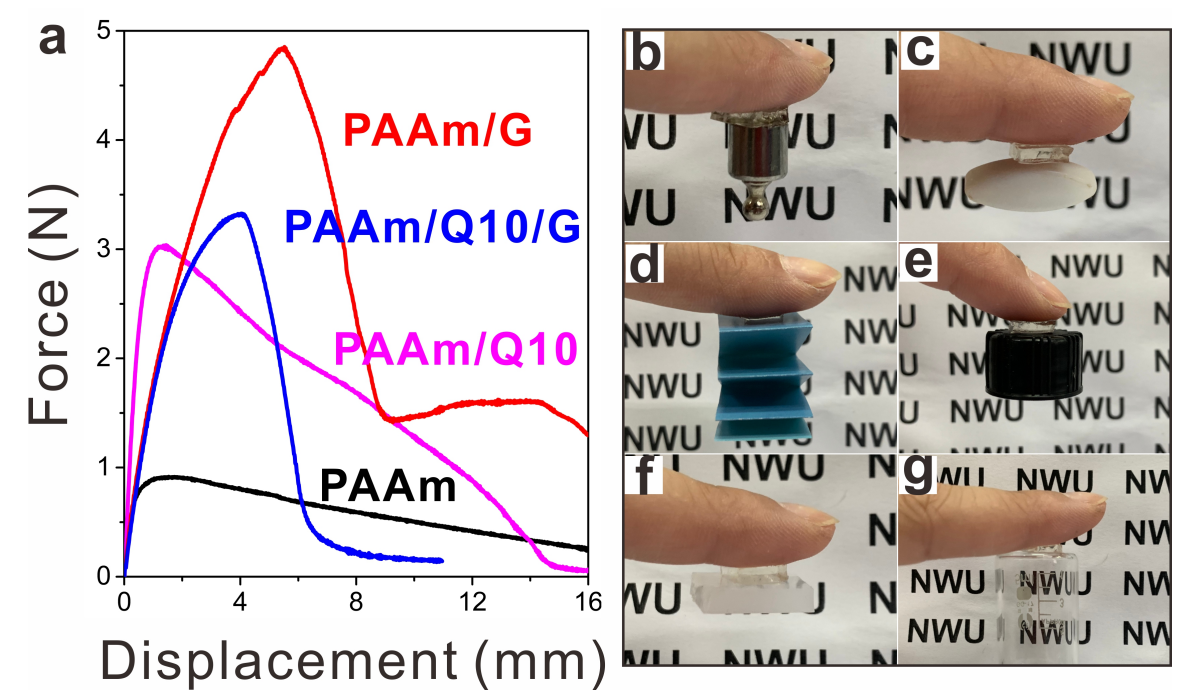

Figure S10. (a) Adhesion tests of different samples. The testing area was $4 \mathrm{~cm}^{2}$. The stretching speed was $50 \mathrm{~mm} \mathrm{~min}^{-1}$. (b-g) Digital images of PAAm/Q10/G hydrogels on different substrates: (b) steel, (c) PTFE, (d) paper, (e) plastic, (f) PDMS and (g) glass.
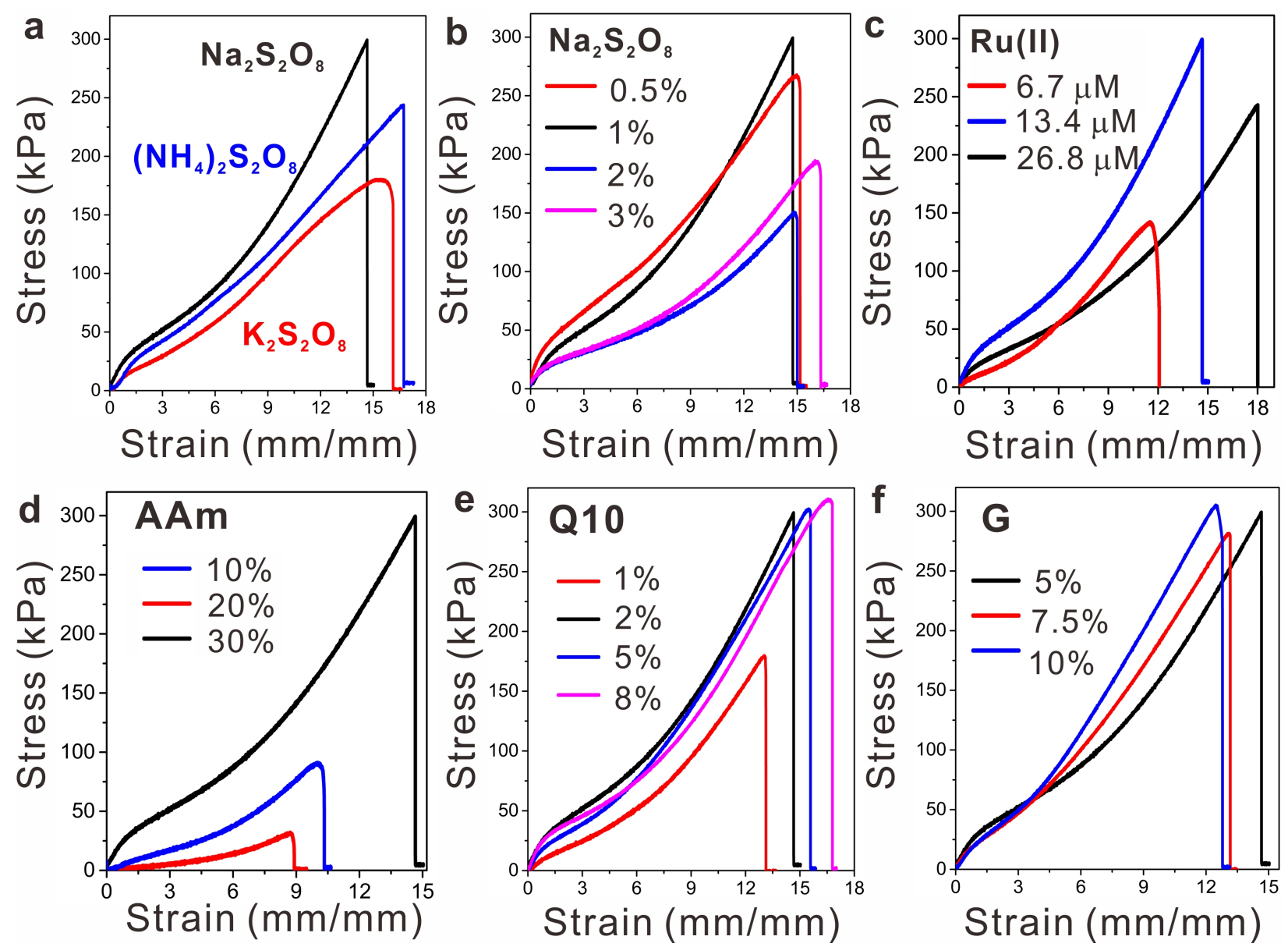

Figure S11. The effects of fabrication conditions on the mechanical property of PAAm/Q10/G hydrogels. All experiments were performed at the following conditions: $[\mathrm{Ru}(\mathrm{II})]=13.4 \mu \mathrm{M},\left[\mathrm{Na}_{2} \mathrm{~S}_{2} \mathrm{O}_{8}\right]=1 \mathrm{wt} \%$, $[\mathrm{AAm}]=30 \mathrm{wt} \%,[\mathrm{MBA}]=0.03 \mathrm{~mol} \%$, [gelatin] $=5 \mathrm{wt} \%,[\mathrm{Q} 10]=2 \mathrm{wt} \%$, and $60 \mathrm{~s}$ irradiation at a intensity of $15 \mathrm{~mW} / \mathrm{cm}^{2}$. For specifically evaluating the effect of preparation conditions on the gelation time, only one concentration was varied, and others were kept constant. The stretching speed was $50 \mathrm{~mm} \mathrm{~min}^{-1}$. 


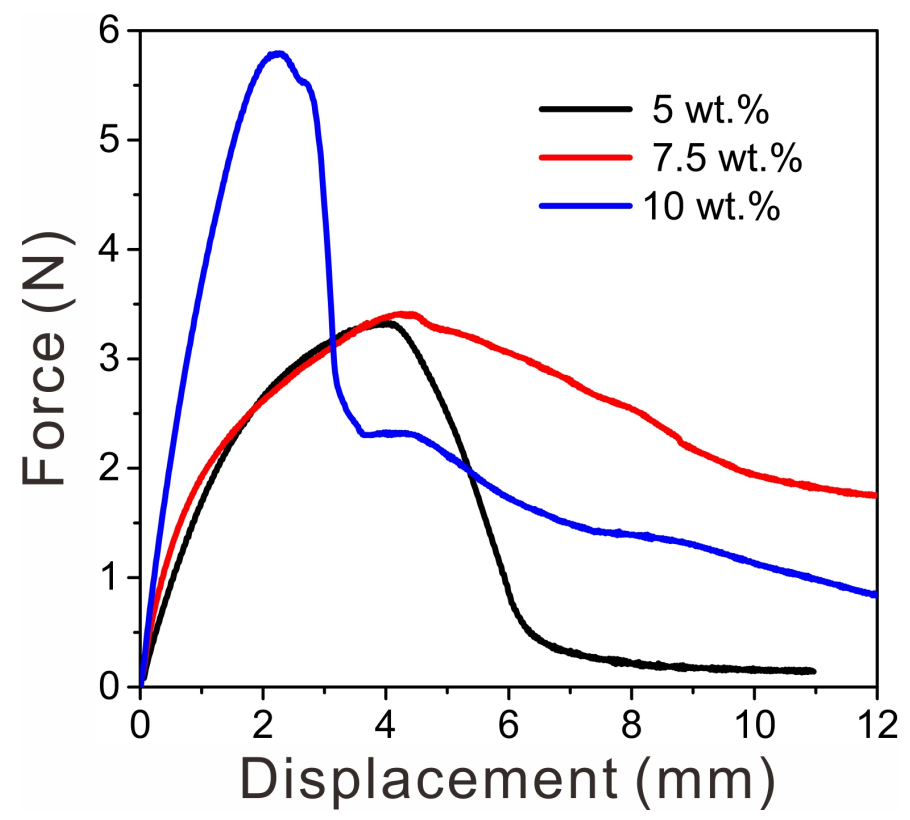

Figure S12. The effect of gelatin content on the adhesion property of PAAm/Q10/G hydrogels.

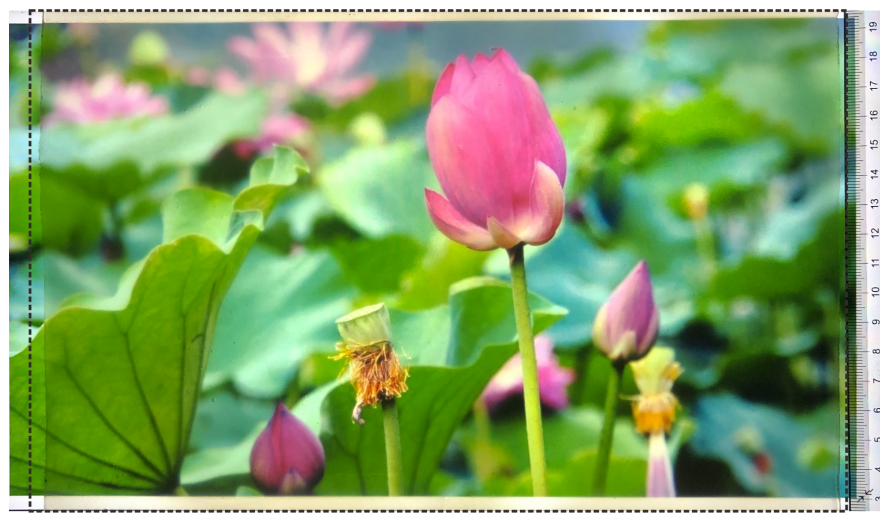

Figure S13. The digital image of transparent PAAm/Q10/G with A4 Size on a picture. Miss Wenqing Xiao took this photograph.
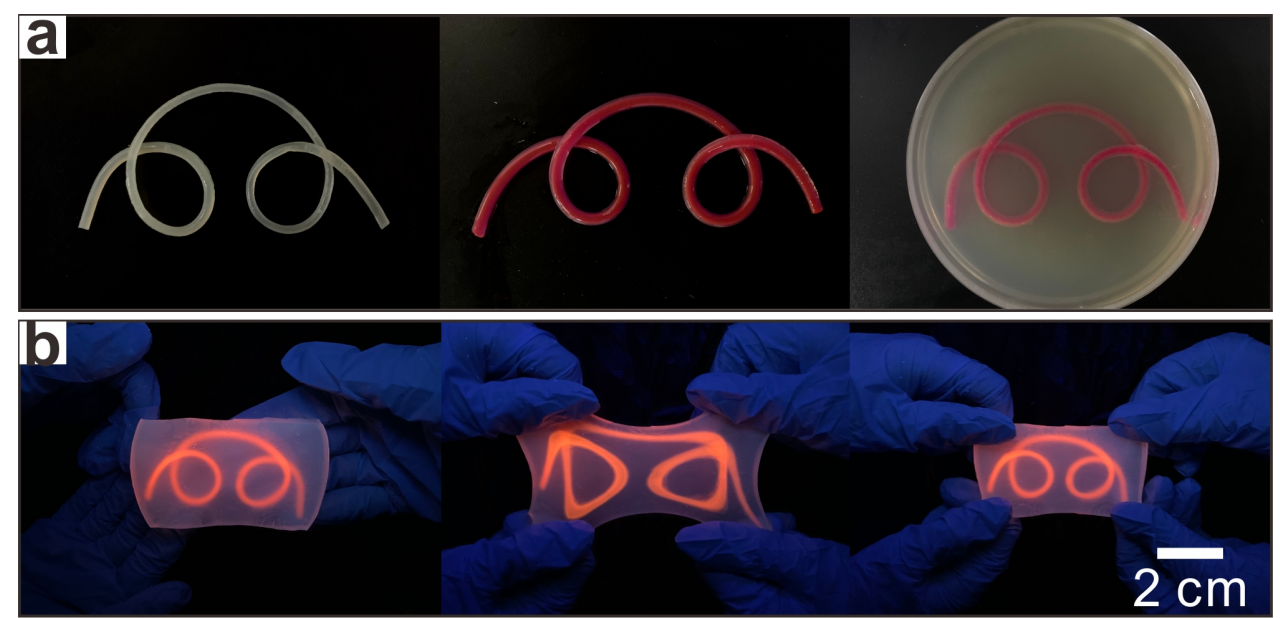

Figure S14. The digital images of (a) fabrication of the fluorescent tube in the bulk hydrogel and (b) the hydrogel at an omnidirectionally stretched state. 


\section{References:}

1. Sun, J.-Y.; Zhao, X.; Illeperuma, W. R. K.; Chaudhuri, O.; Oh, K. H.; Mooney, D. J.; Vlassak, J. J.; Suo, Z., Highly stretchable and tough hydrogels. Nature 2012, 489, (7414), 133-136. 